\title{
New strategy to prevent implant displacement in transaxillary endoscopic augmentation mammaplasty
}

\author{
Sang-Gue Kang ${ }^{1}$, Joung-Ki Kim ${ }^{1}$, \\ Soon Chan $\mathrm{Um}^{2}$ \\ ${ }^{1}$ Department of Plastic and \\ Reconstructive Surgery, Soonchunhyang \\ University Hospital, Seoul; ${ }^{2}$ Um-Nagumo \\ Aesthetic Plastic Surgical Clinic, Seoul, \\ Korea
}

\begin{abstract}
Breast augmentation is one of the most popular cosmetic procedures in Korea and worldwide. In breast augmentation, it is crucial to place the implant in the proper position by creating an adequate pocket considering the size of the implant. This prevents subsequent displacement of the implant. When using an endoscope, the operation is performed without direct vision and in a narrow surgical space, leading to limitations in practice. Inexperienced physicians face a steep learning curve in their efforts to overcome this difficulty. In this study, we attempted to overcome this challenge by specifying the medial and lateral dissection ranges. The extent of the dissection is determined based on the pinnate of the pectoralis major muscle when dissecting medially and the fascia of the pectoralis major, the pectoralis minor, and the serratus anterior muscles when dissecting laterally. In addition, the structure made from the medial and lateral sides can support the implant like a funnel and can prevent further downward, inward, and outward displacement.
\end{abstract}

Keywords Endoscopes / Mammaplasty / Breast implant / Displacement

\section{INTRODUCTION}

Breast augmentation is one of the most popular cosmetic procedures in Korea and worldwide [1]. Until recently, textured implants have been widely used in breast augmentation, as they have been shown to reduce capsular contracture [2]. However, even when using a textured implant, malposition of the implant may occur [3]. Augmentation using an inframammary fold (IMF) incision has the advantage of ensuring that the pockets are more accurately sized, as this allows direct vision while creating the pockets compared to endoscopic surgery. However, Asian patients tend to have more scarring after surgery than Westerners, and in young women, hiding the scar on the axillary area during breast augmentation tends

Received: Jun 23, 2019 Revised: Aug 19, 2019 Accepted: Sep 27, 2019 Correspondence: Sang-Gue Kang Department of Plastic and Reconstructive Surgery, Soonchunhyang University Hospital, 59 Daesagwan-ro, Yongsan-gu, Seoul 04401, Korea

Tel: +82-2-709-9283, Fax: +82-2-796-3543, E-mail: ksps1108@hanmail.net

Copyright $\odot 2020$ The Korean Society for Aesthetic Plastic Surgery.

This is an Open Access article distributed under the terms of the Creative Commons Attribution Non-Commercial License (https://creativecommons.org/licenses/by-nc/4.0/) which permits unrestricted non-commercial use, distribution, and reproduction in any medium, provided the original work is properly cited. www.e-aaps.org to yield high patient satisfaction. Therefore, the endoscopic approach is the most popular approach in Korea [4]. When using an endoscope, the operation is performed without direct vision and in a narrow surgical space, leading to limitations in practice. Inexperienced physicians face a steep learning curve in their efforts to overcome this difficulty. Therefore, we attempted to overcome this challenge by specifying the medial and lateral dissection ranges. The findings of this study indicate that in endoscopic breast augmentation, if the exact medial and lateral edges are checked while performing surgery, a precise pocket can be made, just as in cases of augmentation using an IMF incision.

\section{IDEA}

This method is performed via a transaxillary approach using an endoscopic dissector. The breast width and nipple-to-IMF distance are measured and marked prior to surgery (Fig. 1). The clinician must decide upon the implant to be used and determine the size of the pocket to strictly match the diameter of the implant.

Implant displacement in the upward and downward directions is common. Precise inframammary dissection is important to prevent upward or downward displacement of the implant; however, determination of the lateral and medial pocket size is nearly as impor- 
tant. In particular, if the medial dissection is insufficient, the implant will be displaced, and if the dissection is excessive, it may cause symmastia; thus, intraoperative caution is required. Notably, some reports have argued that the sternal insertion of the pectoralis major muscle should not be cut entirely. Where the pectoralis major muscle stretches to the medial sternal insertion, there are musculotendinous fibers that are finely connected to the rib cage; these fibers are termed the pinnates of the pectoralis major muscle [5]. If these pin-

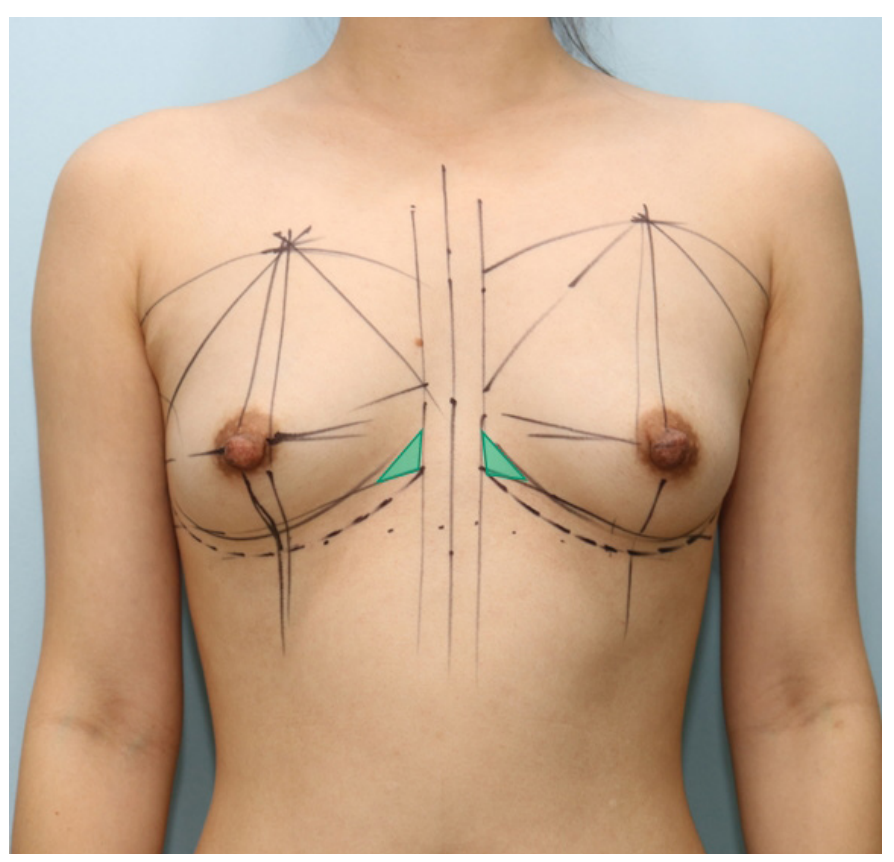

Fig. 1. Preoperative marking (green triangles: medial wedge barrier). nates are finely cut one-by-one, the pocket size clearly grows. The changes in pocket size should be monitored while cutting the proximal pinnate, and when the size of the pocket seems suitable for the size of the implant, dissection should be stopped. This preserves the part of the pectoral muscle that exists between the old and new IMF lines in the inner part of the chest. We termed this the medial wedge barrier. These muscle fibers support the implant such that it does not droop downward, preventing medial malposition (Figs. 1, 2). The medial perforators of the internal mammary artery exit approximately 1 to $1.5 \mathrm{~cm}$ lateral to the midline of the sternum in the subpectoral space [6]. The pectoralis muscle detaches from the midline

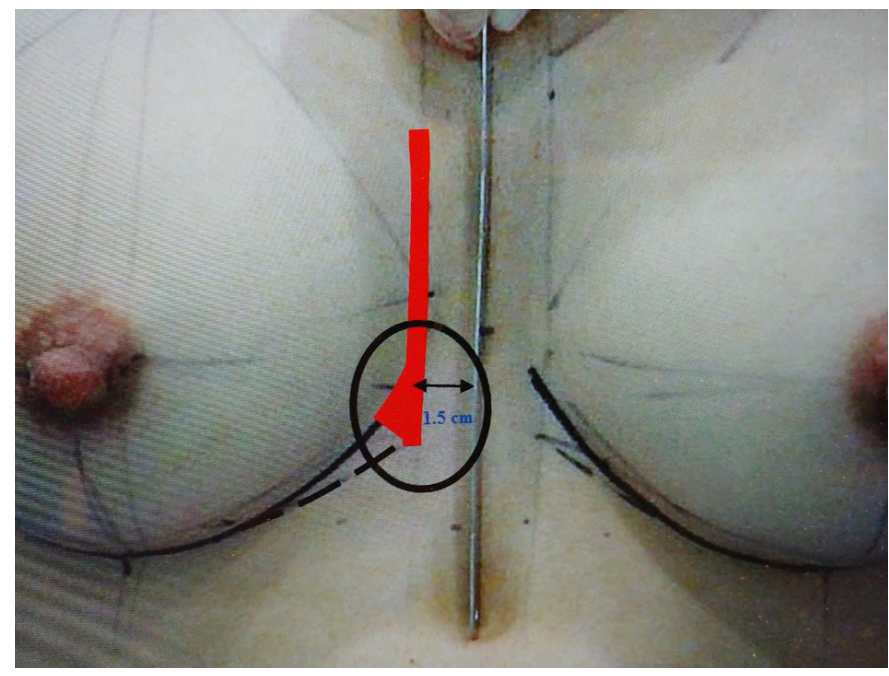

Fig. 3. Picture showing the safe nature of the medial wedge barrier (black circle).
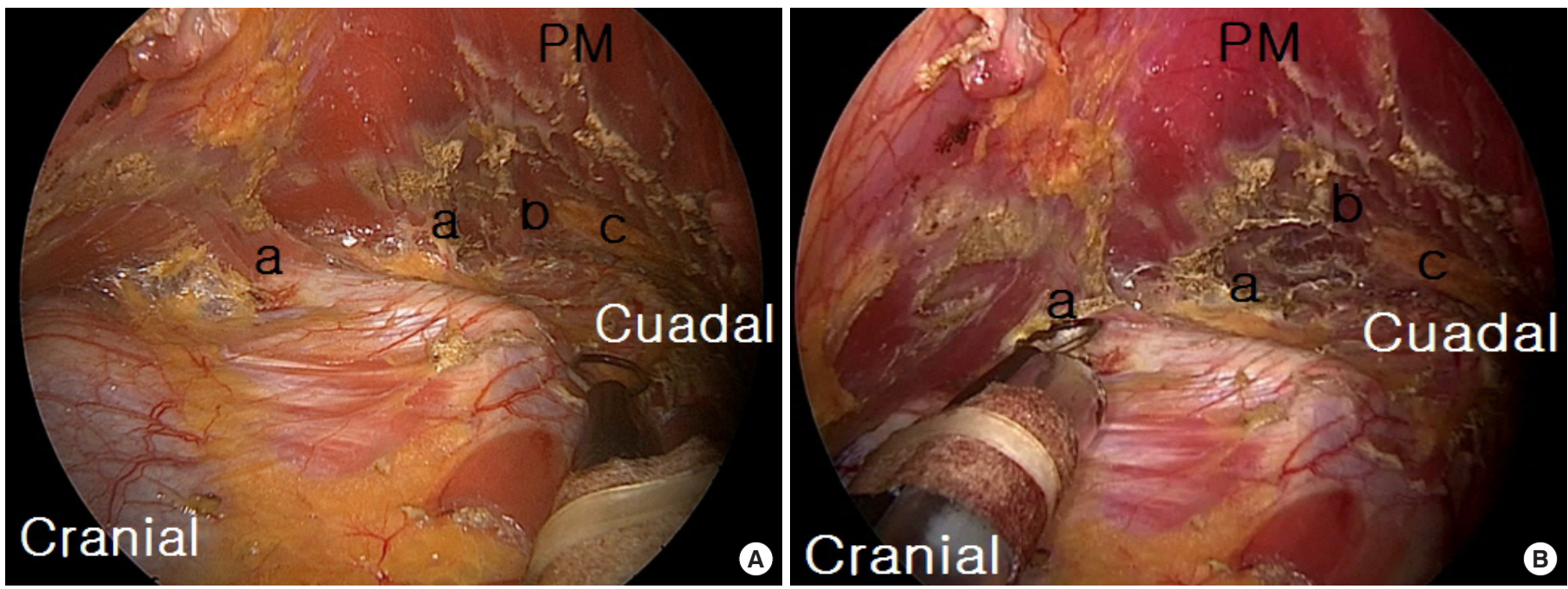

Fig. 2. Triangular wedge-shaped barrier on the medial side. (A) Endoscopic findings of the right breast before dissection. (B) Endoscopic findings of the right breast after dissection. a, proximal pinnate after dissection; b, triangular wedge barrier to be kept; $c$, medial part of new inframammary fold line; PM, pectoralis major muscle. 
1.7 to $1.8 \mathrm{~mm}$ laterally, at the horizontal level of the nipple. Therefore, 2 to $3 \mathrm{~mm}$ is preserved at the site of medial detachment, and a thicker medial barrier is preserved below the nipple (Fig. 3). Thus, the possibility of damage to the medial barrier due to the force of the soft shell of the implant is extremely low. For these reasons, surgery to create a medial barrier is considered safe.

The lateral dissection is also important. If insufficient, the implant will be pushed up, as in cases in which the medial dissection is insufficient. If excessive, the implant will fall laterally. In this regard, Tebbetts [5] suggested that the dissection should be performed only up to the lateral margin of the pectoralis minor muscle. However, we are aware that this recommendation is difficult to follow

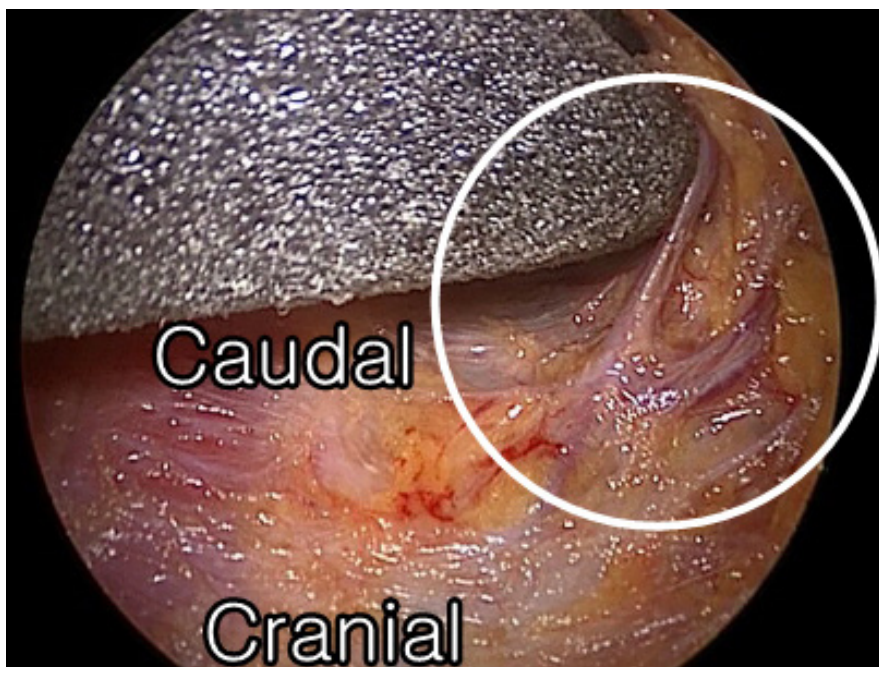

Fig. 4. Picture showing the fourth intercostal nerve in a white circle. in actual clinical practice. Patients increasingly prefer large implants; however, in Asians, the skin is relatively tight and does not stretch well. If we perform dissection in the manner proposed by Tebbetts [5], the result is a pocket that is too small for the implant. Therefore, instead of dissecting beyond the lateral margin of the pectoralis minor muscle, we began performing lateral dissections using a new protocol. Specifically, the boundary of lateral dissection was defined as the point where the pectoralis major, pectoralis minor, and serratus anterior muscles. At this point, the pectoralis minor muscle, serratus anterior muscle, and fourth intercostal nerve (ICN) branch meet; this requires precise dissection (Fig. 4). If the pocket is still smaller than the required size, we can dissect slightly further above the fascia of the pectoralis minor and serratus anterior muscles and adjust the meeting point with the pectoralis major muscle to the lateral side. Thus, we set this point where the deep fascia of the pectoralis major muscle and the superficial fascia of the pectoralis minor and serratus anterior muscles meet as the boundary of the lateral dissection, creating a wall-like boundary. Accordingly, we termed the boundary a lateral solid wall (Fig. 5).

Therefore, the key element of the surgical method presented in this study is that, on the medial side, the implant is placed, as if in a funnel, between the remaining muscle fiber of the pectoralis major muscle (or the deep fascia of the pectoralis major muscle) and the chest wall. On the lateral side, the implant is placed, also as if in a funnel, between the solid wall where the deep fascia of the pectoralis major muscle and the superficial fascia of the pectoralis minor and serratus anterior muscle meet, preventing the implant from moving downward. Furthermore, these structures actually act like a funnel, which can prevent implant displacement to the medial and lateral sides (Fig. 6).
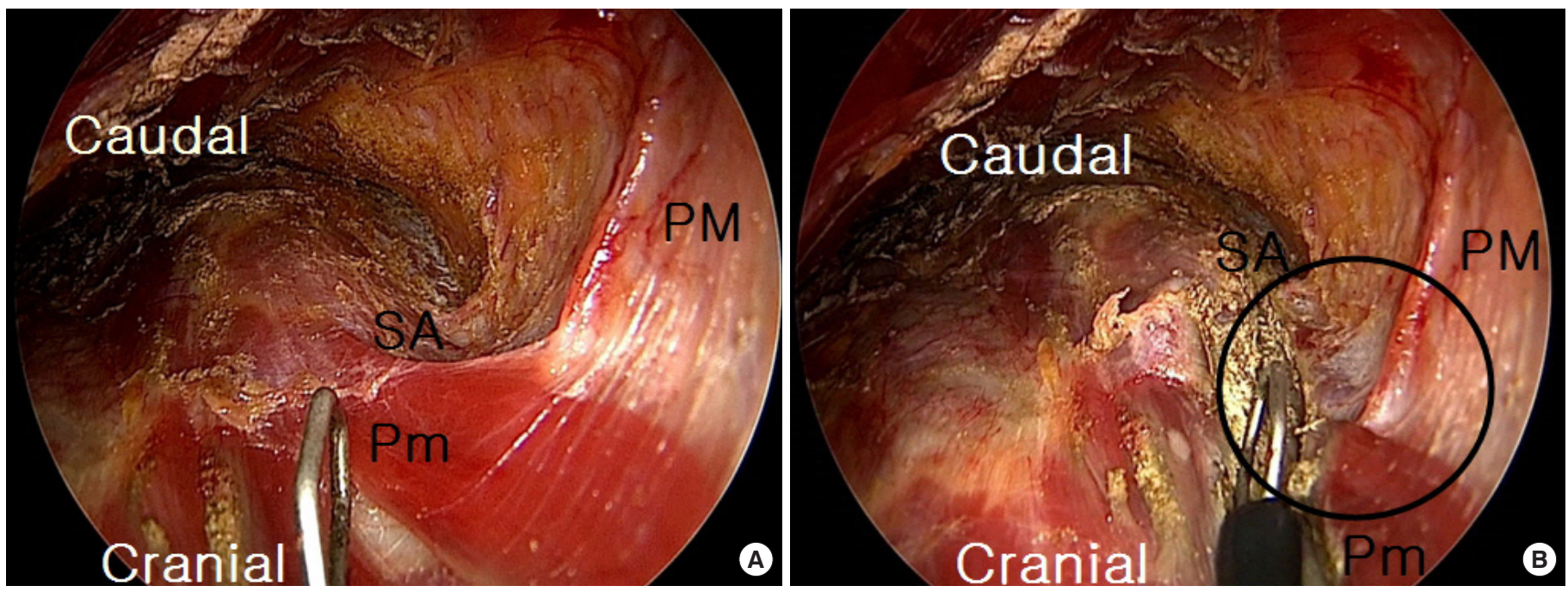

Fig. 5. Solid wall on the lateral side. (A) Endoscopic findings of the right breast before dissection. (B) Endoscopic findings of the right breast after dissection. More space became available after dissection in the area marked by a black round circle. PM, pectoralis major muscle; Pm, pectoralis minor muscle; SA, serratus anterior muscle. 


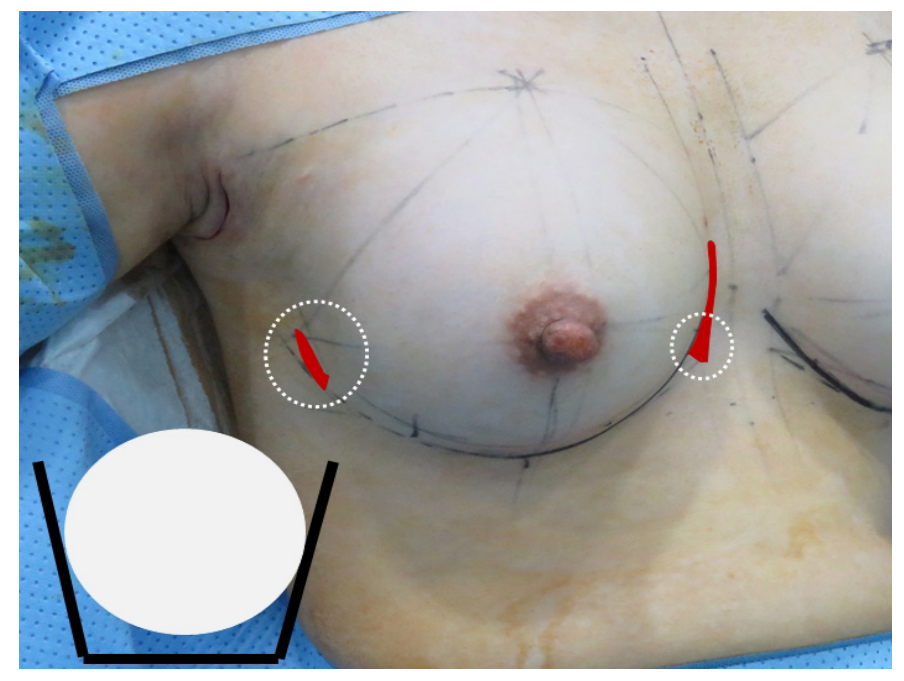

Fig. 6. Both barriers (white dotted circles) hold the implant. The implant is prevented from moving downward, as if caught in a funnel.

\section{DISCUSSION}

The transaxillary approach to breast augmentation was introduced in the 1970s. At that time, most plastic surgeons did not pay attention to this approach, despite its effectiveness as a surgical method. Since the operation was performed via blunt dissection and was unfamiliar, most surgeons neglected the procedure [7,8]. Displacement of the implant occurred in $8.6 \%$ of cases using blunt dissection [9]. However, in 1993, Ho [10] used endoscopy during breast augmentation, and this led to the possibility of operation under direct vision. As a result, meticulous dissection, instead of blunt dissection, became possible, and the transaxillary approach became popular. Asian patients tend to have more scars after surgery than Westerners, and in young women, hiding the scar on the axillary area during breast augmentation yields high patient satisfaction. Therefore, the endoscopic approach is the most popular approach in Korea [4].

Endoscopic breast augmentation often results in asymmetry of the IMF line and displacement of the implant. To solve this problem, plastic surgeons use various methods. Moliver et al. [11] reported that medial displacement of the implant occurred due to excessive muscle fibers in the pectoralis major or pectoralis minor muscle. Therefore, they insisted that sufficient dissection be performed to prevent the medial displacement of the implant. Our method is similar to the above theory in that it involves dissection of the fascia of the pectoralis major, the pectoralis minor, and the serratus anterior muscle and that it leaves the lateral wall to fit the size of the implant. Sim and Sun [12] stated that round-base and full-height anatomical implants provide greater stability. They reported that the selection of these implants reduces the rotation and displacement of the implants, and they suggested another method, in which an implant pocket was made using the subfascial layer. The dissection level at the inferior margin was at the subfascial plane, and the split fascia on the muscle was expected to contribute to limiting the inferior migration of the implant. However, only a limited number of patients can use this method (as determined by using a pinch test result of $<3 \mathrm{~cm}$ at the upper pole of the breast and $<4$ $\mathrm{mm}$ at the IMF level) [13].

Surgery using a transaxillary incision results in more displacement of the implant than using an IMF incision [14]. To reduce implant displacement, we devised our method, which focuses more on accurately adjusting the size of the implant when creating new pockets than on manipulating the IMF area. This concept may be appropriate for macrotextured implants. When the operation is performed using our method, the pocket is formed and exactly matches the diameter of the implant. In addition, the medial and lateral dissection is performed such that the implant falls sufficiently inferiorly and is positioned properly. Because the implant is held in the shape of a funnel, the triangular wedge barrier on the medial side and the solid wall on the lateral side can also prevent downward displacement of the implant, even if the dissection is performed below the IMF line. This can reduce the incidence of postoperative seroma and hematoma by eliminating dead space. In addition, the wedge barrier reduces the incidence of symmastia, and the lateral solid wall that is retained during the lateral dissection allows preservation of the fourth ICN, resulting in the preservation of the sensory nerve to the nipple or chest wall.

Recently, a sixth-generation implant, the microtextured implant, was launched. Microtextured implants have the characteristics of smooth implants, unlike macrotextured implants. In cases in which a microtextured implant is used, implant displacement frequently occurs [15]. However, with our method, we use a larger implant than the pocket that we create. This minimizes the displacement of the implant. Placing an implant that is too large compared to the pocket may cause rippling; thus, the implant should be approximately one size larger than the pocket, which is thought to be sufficient to prevent later rippling due to drooping of the skin. Using this method, the surgical technique introduced in this study can be applied when using microtextured implants.

The main limitation of our method is that the size of the pocket must be determined precisely, with the size of the implant in mind. Some surgical experience is required to perform this technique. Additionally, because the implant is flexible, it may be displaced downward through the medial and lateral walls. However, we expect that less displacement will occur than when using conventional methods because the fascia and tissues of the IMF can support the implant.

\section{NOTES}

\section{Conflict of interest}

No potential conflict of interest relevant to this article was reported. 


\section{Ethical approval}

The study was performed in accordance with the principles of the Declaration of Helsinki.

\section{Patient consent}

The patient provided written informed consent for the publication and the use of her images.

\section{ORCID}

$\begin{array}{ll}\text { Sang-Gue Kang } & \text { https://orcid.org/0000-0001-8055-6591 } \\ \text { Joung-Ki Kim } & \text { https://orcid.org/0000-0002-7882-0522 } \\ \text { Soon Chan Um } & \text { https://orcid.org/0000-0002-2383-1523 }\end{array}$

\section{REFERENCES}

1. Cosmetic Surgery National Data Bank Statistics. Aesthet Surg J 2017; 37(suppl_2):1-29.

2. Brohim RM, Foresman PA, Hildebrandt PK, et al. Early tissue reaction to textured breast implant surfaces. Ann Plast Surg 1992;28:354-62.

3. Khanna J, Mosher M, Whidden P, et al. Reoperation Rate after Primary Augmentation with Smooth, Textured, High Fill, Cohesive, Round Breast Implants (RANBI-I Study). Aesthet Surg J 2019;39:1342-9.

4. Sim HB. Transaxillary endoscopic breast augmentation. Arch Plast Surg 2014;41:458-65.

5. Tebbetts JB. Dual plane breast augmentation: optimizing implant-softtissue relationships in a wide range of breast types. Plast Reconstr Surg 2001;107:1255-72.
6. Siggelkow W, Lebrecht A, Kolbl H, et al. Dual-plane implant positioning for capsular contracture of the breast in combination with mastopexy. Arch Gynecol Obstet 2005;273:79-85.

7. Tebbetts JB. Transaxillary subpectoral augmentation mammaplasty: long-term follow-up and refinements. Plast Reconstr Surg 1984;74: 636-49.

8. Wright JH, Bevin AG. Augmentation mammaplasty by the transaxillary approach. Plast Reconstr Surg 1976;58:429-33.

9. Howard PS. The role of endoscopy and implant texture in transaxillary submuscular breast augmentation. Ann Plast Surg 1999;42:245-8.

10. Ho LC. Endoscopic assisted transaxillary augmentation mammaplasty. Br J Plast Surg 1993;46:332-6.

11. Moliver CL, Sanchez ER, Kaltwasser K, et al. A Muscular etiology for medial implant malposition following subpectoral augmentation. Aesthet Surg J 2015;35:NP203-10.

12. Sim HB, Sun SH. Transaxillary endoscopic breast augmentation with shaped gel implants. Aesthet Surg J 2015;35:952-61.

13. Sim HB. Revisiting prepectoral breast augmentation: indications and refinements. Aesthet Surg J 2019;39:NP113-22.

14. Namnoum JD, Largent J, Kaplan HM, et al. Primary breast augmentation clinical trial outcomes stratified by surgical incision, anatomical placement and implant device type. J Plast Reconstr Aesthet Surg 2013; 66:1165-72.

15. Huemer GM, Wenny R, Aitzetmuller MM, et al. Motiva ergonomix round silksurface silicone breast implants: outcome analysis of 100 primary breast augmentations over 3 years and technical considerations. Plast Reconstr Surg 2018;141:831e-842e. 